\title{
Case report: Delayed diagnosis of Addison's disease and Autoimmune Polyglandular Syndrome Type 2 due to misinterpretation of short synacthen test
}

I Teoh, H Rathur

Tameside General Hospital, Fountain Street, Ashton-under-Lyne OL6 9RW

\section{Background}

- Short Synacthen test (SST) is commonly used to confirm diagnosis of Addison's disease.

- However, misinterpretation can happen in inexperienced hands and this can lead to potentially adverse outcome.

- We present a case in which a diagnosis of Addison's disease was missed as his SST was misinterpreted due to background hydrocortisone injection being administered to the patient.

- This patient was also found to have Autoimmune Polyglandular Syndrome Type 2 (APS-2) after further tests were performed.

\section{Case Study}

- A normally fit and well 28-year-old Caucasian man presented to hospital with a few days history of general malaise and a syncopal episode.

- On admission, patient was hypotensive and tachycardic.

- Admission bloods showed hyponatraemia, hyperkalaemia, acute kidney injury and raised inflammatory markers.

- The diagnosis of Addison's disease was suspected.

- Patient was given hyperkalaemia treatment, intravenous fluids, antibiotics and hydrocortisone.

- Patient markedly improved over the next few hours.

- On the day after, SST was performed without holding off patient's morning dose of hydrocortisone.

- Therefore, his SST results showed high cortisol levels.

- This was misinterpreted as ruling out adrenal deficiency.

- He was hence discharged without hydrocortisone replacement.

- Two weeks later, patient was re-admitted to hospital with similar presentation.

- SST was repeated before patient's morning dose of hydrocortisone.

- This time, it demonstrated flat response and finally confirmed patient's diagnosis of Addison's disease

Patient was started on oral hydrocortisone and fludrocortisone.

Patient's blood test also showed evidence of autoimmune hypothyroidism.

- Further blood tests were performed to screen for other conditions associated with APS-2.

Patient was also found to have probable underlying pernicious anaemia.

\section{Investigations}

\begin{tabular}{|ll}
\hline First admission & \\
\hline Cortisol (on admission) & 164 \\
\hline 9am Cortisol (before ACTH injection) & 1655 \\
\hline Cortisol (30 mins after ACTH injection) & 1655 \\
\hline Sodium & 127 \\
\hline Potassium & 6.7 \\
\hline Urea & 23.3 \\
\hline Creatinine & 319 \\
\hline CRP & 206 \\
\hline
\end{tabular}

\section{Cor}

\section{$\mathrm{P}$}

